\title{
A three-step scheduling based on geographic information system for workforce- planning problem
}

Sinthamrongruk, Thepparit; Dahal, Keshav; Wang, Xinheng; lqbal, Muddesar

Published in:

Proceedings of the 2019 13th International Conference on Software, Knowledge, Information Management and Applications (SKIMA)

DOI:

10.1109/SKIMA47702.2019.8982422

Published: 06/02/2020

Document Version

Peer reviewed version

Link to publication on the UWS Academic Portal

Citation for published version (APA):

Sinthamrongruk, T., Dahal, K., Wang, X., \& Iqbal, M. (2020). A three-step scheduling based on geographic information system for workforce-planning problem. In Proceedings of the 2019 13th International Conference on Software, Knowledge, Information Management and Applications (SKIMA) (IEEE Proceedings). IEEE. https://doi.org/10.1109/SKIMA47702.2019.8982422

\section{General rights}

Copyright and moral rights for the publications made accessible in the UWS Academic Portal are retained by the authors and/or other copyright owners and it is a condition of accessing publications that users recognise and abide by the legal requirements associated with these rights.

Take down policy

If you believe that this document breaches copyright please contact pure@uws.ac.uk providing details, and we will remove access to the work immediately and investigate your claim. 
“(C) (C) 20xx IEEE. Personal use of this material is permitted. Permission from IEEE must be obtained for all other uses, in any current or future media, including reprinting/republishing this material for advertising or promotional purposes, creating new collective works, for resale or redistribution to servers or lists, or reuse of any copyrighted component of this work in other works." 


\section{A three-step scheduling based on geographic information system for workforce-planning problem}

\author{
Thepparit Sinthamrongruk \\ University of the West of Scotland \\ Paisley, United Kingdom \\ thepparit.sinthamrongruk@uws.ac.uk \\ Xinheng Wang \\ University of West London \\ London, United Kingdom \\ henry.wang@uwl.ac.uk
}

\author{
Keshav Dahal \\ University of the West of Scotland \\ Paisley, United Kingdom \\ keshav.dahal@uws.ac.uk \\ Muddesar Iqbal \\ CIP Ltd \\ United Kingdom \\ m.iqbal@ciptechnology.co.uk
}

\begin{abstract}
Routing workforce planning problem is a difficult problem, requiring a combination of the vehicle routing problem and the staff scheduling problem. This problem associates with a lot of constraints, even when having only a single criterion and homogeneous staff skill. This paper introduces three-step scheduling-a generic scheduling procedure for solving the workforce planning problems which incorporates between an optimisation tool, fuzzy inference system and geographic information system technology. The scheduling problem is hierarchically divided into sub-problems and then solved with the proposed scheduling framework. A simulated case study of the electricity meter installation service problems is employed to verify the proposed framework. In this study, we are focusing on the route scheduling which is the first step of the three-step scheduling. The total of travel distance is defined as an objective function. Particular case requirements such as lunch and parking time are also included in the model for optimisation. Our empirical study reveals that the proposed route scheduling is capable of exploring the optimal solutions for the case study with the shortest service path, compared to the original procedure.
\end{abstract}

Keywords-workforce planning problem; scheduling procedure; route scheduling; genetic algorithm; electricity meter installation

\section{INTRODUCTION}

Smart meters are the next generation of electricity and gas supply meters to offer a range of intelligent functions such as how much energy the customers are using. The smart meters also communicate directly with the energy supplier to save time and budget for visiting the customer home to read and confirm energy usage. Meanwhile, customers can manage energy use and reduce unnecessary energy consumption which saves pollution emissions [1]. Thus, the demand for meter installation has growth, especially, in the winter which customer would like to control the energy bills under their restrained budget.

Electricity meter installation (EMI) problem is one of workforce planning problems focusing on how to create a service schedule for engineers for meter installation. This issue requires a combination of the vehicle routing problem (VRP) and the workforce-planning problem which associates with many constraints, even when having only a single criterion and homogeneous staff skill. This problem concentrates on when and how engineers should be employed and when skills are incorporated into a model, which decides under a specific time window.

Even the procedure of the EMI problem might vary depending on each energy supplier relating to various conditions, i.e., the size of the service area, and public or private service or regulatory settings. Fig. 1 illustrates an example of a general overview of the EMI problem where there are eight customers, from Customer-A to Customer-H, who require installing the smart meter. In general, the procedure of the service can be summarised as follows:

- Firstly, customers register and inform the intention to install the smart meter on the website/chat box.

- The energy supplier checks and confirms the right of the customer.

- Then, the meter operator team plans the sequence of service routes considering the customer information collected as well as the condition of transportation

- The meter operator team sends customers a letter or an email to inform an appointment for installing the meter.

- If there is no problem on the appointment date, the engineers are assigned to install the new meter at customer's homes regarding the planned schedule.

- Lastly, each engineer starts their journey from the office; and terminates the mission at the office after service activities are completed.

From experience, the energy supplier has received higher demand for the meter installation than normal especially, in the winter season. The planner is unable to schedule the job properly with the minimising cost that brings difficulties and challenges for the planner and the service supplier for management and scheduling. Thus, a practical effective service route with precise transportation time and distance, so minimising operation costs is essential.

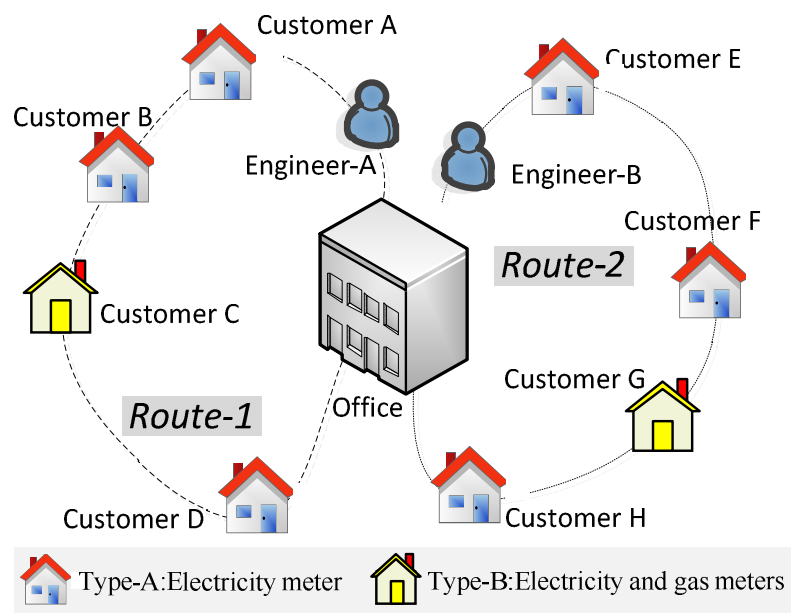

Fig 1. Overview of workforce planning problem 
In this paper, we apply a generic three-step scheduling framework for solving workforce planning problems of the EMI. The highlight of this framework is in solving the problem from a hierarchical perspective depending on high-low priority by dividing the problem into sub-problems: (i) route scheduling, (ii) resource selection and (iii) local improvement and then finding solutions with priority (i) - (iii). The designed structure integrates the meta-heuristic algorithm using a genetic algorithm(GA)-based approach incorporating with fuzzy logic system and GIS technology for solving reliable and practical problems. With the proposed approach, it guarantees that the total travelling distance is minimised under the timeslot windows. To verify that the proposed framework, the scheduling problem of the EMI service is applied to explore the experimental results.

The remaining part of this paper is laid out as follows. Section-2 presents the workforce planning scheduling problem. Section-3 explains the proposed techniques and approach. Section-4 provides the case studies and the experimental results. Section-5 presents the conclusion and the direction for future works.

\section{RELATED WORK}

The workforce planning in a company is one of the most difficult problems managers face [2]. In general, the solution for solving the workforce planning problem can be categorised into two directions: 1) managerial studies which gives an extensive logical description and 2) mathematical model or technical perspective which focuses on very hard mathematical models neglecting the real implication.

Regarding the managerial aspects, the variant skill of workers is considered. The performance, more experienced worker can finish the job faster than the workers with a lower skill. This is referred to as substitution or selection when the schedule is unexpectedly changed. The service provider can use the skill factor to decide whether or not to assign a job for the workers. However, the operating time in different experience has less affect for the operation in the jobs/services controlled by the strict policy/procedure. Concerning the wage, higher skilled workers may involve higher labor costs as they can increase the speed of the work. However, it is not clear that higher skill workers get higher wage than lower skill worker. In studies of $[3,4]$, the models do not concern difference in the wages. In real operation, unexpected changes in the demand for services is difficult to know. The planner is motivated to produce the best solution that can offer a flexible service with the minimised operational budget. With this issue, a variety of relevant methods for solving the workforce planning problem have been proposed for exploring the best service taking into account the stochastic nature of the problem. For example, a fuzzy set theory was integrated for planning and scheduling to approximate the potential performance of the worker [5]. Burke et al. [6] reviewed and overviewed papers between 1990s until 2004 related nurse rostering problem and revealed that meta-heuristics such as genetic algorithm (GA) and simulated annealing (SA) are promising for very difficult real world problems for which optimal solutions cannot be obtained with exact approaches. Simultaneously, there is a trend to apply chaining and merging procedure for data analysis as an attempt to pass the output of the previous method to be an input of the next process while the former technique combines a summary of the results deriving from different methods. However, the best way for solving the high demand problem is to employ an effective scheduling technique with a rapid, precise and reliable feature to be ready for re-scheduling when demand or constraints change daily.

The workforce planning problem can be referred as the VRP with time windows (VRPTW). The conventional objective mainly aims minimising the travel distance and the transportation cost [7] and service time consisting of the total travel time, waiting time, and total travel distance are defined as hard constraints of the problems. To solve the VRPTW, the Travelling Salesman Problem (TSP) is employed to represent the form of the VRPTW [8]. Due to the combination of the complexity of the modern problems, the multiple travelling salesman problem (MTSP) has increasingly received intensive attention for the VRPTW. The MTSP associates with $m$ salesmen, a set of $\mathrm{n}$ task-locations to visit where $m>n$ with each task location visited exactly once; while TSP involves only one salesman. The objective of the MTSP is to minimize the sum of distances travelled by each salesman [9]. For the time-invariant, this feature has been mentioned in the majority of studies relating to solving the routing problems [10]. Service time can be affected due to various factors such as traffic congestion, weather condition, and traffic restrictions in practical situations. The time-dependent model also plays a major role in a variety of the VRP arising in distribution planning, garbage collection, the EMI service etc. These can vary the quality of the optimising solution.

In this paper, we apply a generic scheduling framework for solving the EMI service that considers the issues and theories mentioned above. Further details and implementation are demonstrated in the following sections.

\section{THREE-STEP SCHEDULING BASED ON GIS}

The early research has applied a simple or single method for addressing workforce-scheduling problems. However, single meta-heuristics method cannot tackle the dynamic and uncertain nature of modern problems [6]. In light of the rapid development of computing science, many of the more complex and hybrid optimisation methods have been proposed for solving the scheduling problem. In this paper, we propose to consider these concepts to develop a three-step scheduling.

\subsection{The three-step scheduling}

The structure of the three-step scheduling is designed with the notion that the quality of the travel distance is the most important priority; significantly contributing to the quality of the service plan and operational cost. The whole problem is hierarchically divided into sub-problems based on the VRP and the scheduling problem, and then solving the problem with three steps: step-1) route scheduling, step-2) resource selection, and step-3) local improvement. So too, one of the advantages of three-step scheduling is that this framework extracts explicitly the sub-problems that are of practical 
importance for each particular problem to allow additional constraints. This scheduling framework integrates a component of a highly-reliable travel distance between locations obtained from GIS for optimising the travel distance in real-world scenarios.

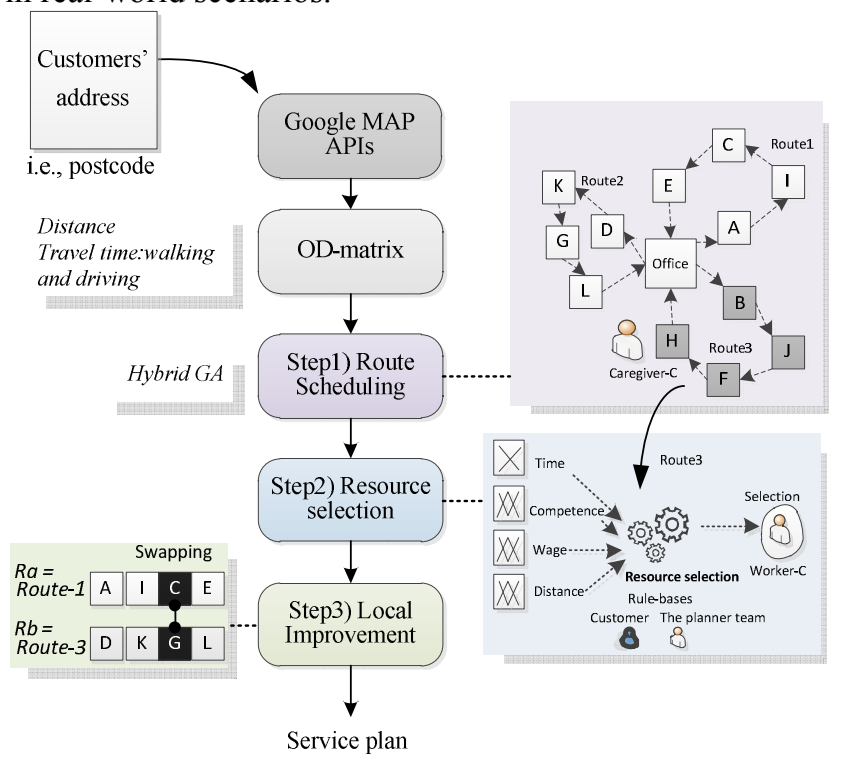

Fig 2. The overview of a three-step scheduling

Fig 2. illustrates the overall structure of the proposed threestep scheduling. Further details of each will be described in the following:

Step1-Route scheduling focuses on how to arrange the solution of the service route while minimising the total of distances travelled for $r$ routes containing $n$ tasks' locations, taking both the limitation of workers and constraints of time windows into consideration. The sub-problem is defined as MTSP and then employing a hybrid GA-based algorithm - a metaheuristic approach to optimise the model, and achieve an optimal service route. We select the GA to integrate as into the model for two reasons: 1) GA is capable of exploring the optimal global solution with a high probability of success. 2) GA has been applied for large-scale optimisation problems as a suitable technique.

In addition, the system is designed for supporting the practical modern scheduling problems. High accuracy data such as precise transportation times and distances in different modes of transportation are used in the proposed model through integration of the Google Maps-the most popular GIS technology. The daily uncertain conditions/constraints for scheduling, i.e., increasing traffic jam, particular time to limit vehicle speed, or a schedule for swapping between a two-way and a one-way road is obtained from the Google to create an origin-destination (OD) matrix instead of using a conventional method for evaluating the distance and travel time before the hybrid GA started. The procedure of the proposed GA for solving the workforce planning system is presented in Algorithms 1.

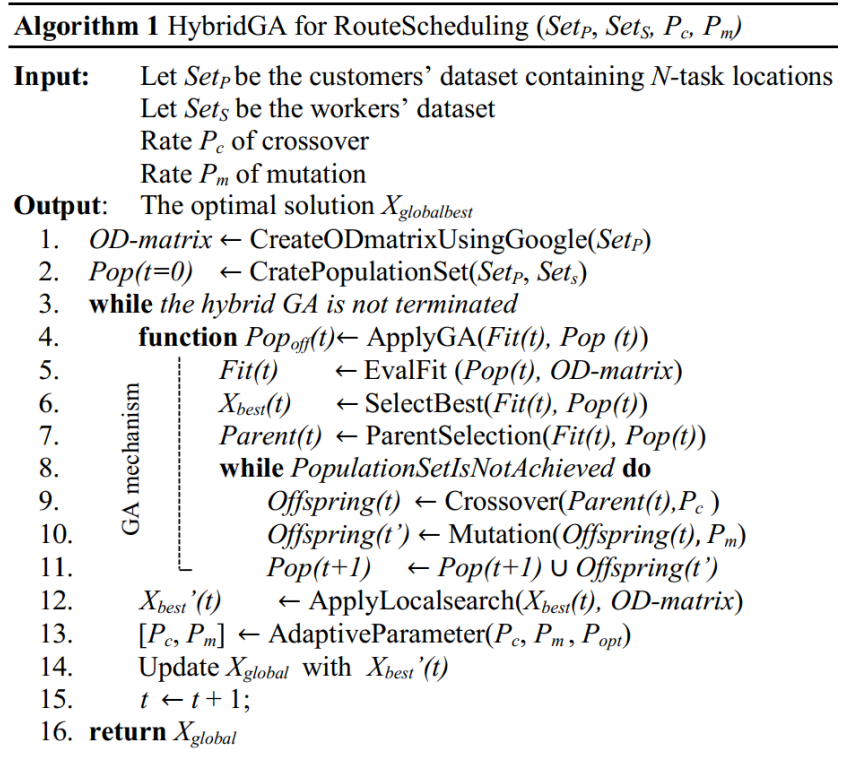

Given $t$ is the generation number of the hybrid GA, the $O D$ matrix is created using a Google MAP APIs in line 1. The mechanism of the hybrid GA starts from line 2 by creating a set of population. Inside the while-loop, the EvalFit function evaluates the sum of travel distances and returns the fitness values Fit $(t)$. The SelectBest selects the parents' chromosomes from the set of populations depended on Fit $(t)$. The best solution Xbest of each generation is stored in line 6. Lines 8 to 11 provide the mechanism of GA operators for generating offsprings or Pop $(t+1)$ for next generations. The local search is applied to enhance Xbest in line 12 while line 13 presents a procedure of adaptive GA to adjust the crossover probability $\left(P_{c}\right)$ and mutation probability $\left(P_{m}\right)$. The procedure in line 14 compares/updates improved solution Xglobal. The algorithm repeats line 3 to 15 until the number of generations reaches the defined number. Finally, an optimal service route is generated under constraints.

Step2-Resource selection aims to match workers to each service routes and jobs. The designed structure integrates the heuristic search and fuzzy logic to develop a reliable and practical decision to match qualified each worker to each operation. The resource selection system is firstly responsible for handling all information and data, such as retrieving the planned route from the hybrid GA in stepl, customers' and workers' profiles; and then matching between the correct workers and customer tasks. The operational cost is subsequently minimised, considering preference and possible time windows. Then, the system employs fuzzy logic with rulebases acquired from both customers and the planner team to create fuzzy inference system (FIS) to estimate a set of scores for all workers in each task. One of the advantages of the resource selection step is that the system is designed to address a particular case requirement for multiple-engineers by using a decision from the fuzzy inference system. Fig. 3 illustrates the structure of FIS with four input variables, which are defined as membership function consisting of 1) Time: low and high, 2) 
Distance: near, average, and far, 3) Competence: low, average, high, and 4) Cost: save, average and high.

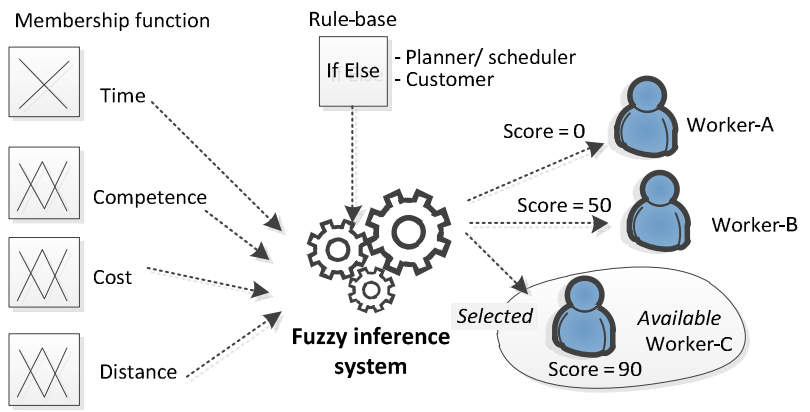

Fig. 3. FIS for match engineers to service routes and handling with the requirement of multiple engineers

Step3-Local improvement focuses on 1) exploring the best solution of Step 2 and 2) re-checks and repairs the service plan generated by the previous steps in case of infeasible solution using the swapping procedure. This step interchanges activities between two engineers' routes. Given each route has a set of the sequence of service activities, the process starts by selecting a pair of two routes $R a$ and $R b$ and swapping the service activities at position $c$ along the chosen pair. The service plan after re-arrangement is recalculated with the objective function evaluation. If an improvement is found, the new service plan is proposed.

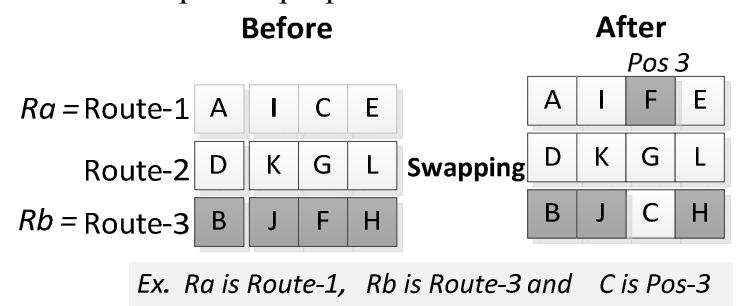

Fig. 4. Local improvement

\section{CASE STUDIES}

In this paper, we are focusing on the route schedule for the EMI service problems to verify the proposed technique. The following sections provide the problem details and experimental results.

\subsection{The EMI service problem}

Energy efficiency belongs at the heart of a low-carbon economy. In a typical UK household, more than half the money spent on fuel bills is for heating and hot water. Smart meters are the next generation of electricity and gas meters, which offer a range of intelligent functions [1]. Unlike its predecessor, the smart meters can assist the customer to keep track of the energy consumption in the household and will cut out the need for meter readings as well as report real-time information about energy use. For installation, the customer will not be charged separately for a new smart meter. In addition, the customer can get the best offers from energy companies i.e., fixed price rate for the winter season. Thus, the demand for the smart meter installation is higher volumes than normal especially in the winter season due to the customer need to manage their energy use. The procedure for meter installation begins with receiving the intention from customers. Then, the meter installation team will plan the schedule for an engineer going to install the smart meter at customers' home. When the engineer runing late or cannot complete daily assigned tasks, they are responsible to inform the delay and cancellation of the service to the next customer by themselves. Thus, the engineers face with risk of the customers complaint when the appointment is delayed or cancelled.

The following section describes the details for the EMI service

- Each engineer is recommended to work a daily service at 5 jobs.

- Engineers earn a fixed rate of wage at 30 pounds per job.

- Each customer has the same priority which cannot demand specified service time.

- Each engineer starts working at the office at 08:00 and is recommended to finish the last job between 16:30 to 18:30.

- Engineer enables to terminate their work after last job finished. Procedure is not required engineers going back to the office.

- There are two types of meter installation: Type- $A$ ) electricity meter and Type- $B$ ) both electricity and gas. Installation time is set at the default 90 minutes for Type- $A$ and it increases up to 180 minutes for Type-B.

- Concerning skill and competence, each engineer enables to install both types of smart meter installation with the same competence.

\subsection{Objective function, constraints and parameter setting}

We give the mathematical notations of the model:

$$
\begin{aligned}
& N=\{0,1,2,3, \ldots, n, n+1\} \text { : The set of task-locations on the map, which includes }
\end{aligned}
$$

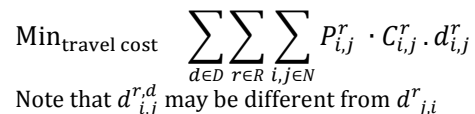

$$
\begin{aligned}
& \text { Subject to } \\
& P_{i, j}^{r, d}=\left\{\begin{array}{c}
1, \text { if a journey is made from } \text { location }_{i} \text { to } \text { location }_{j} \\
0, \text { otherwise }
\end{array}\right. \\
& \sum_{r \in R} \sum_{i, j \in N} P_{i, j}^{r}=1 \quad \forall r \in R \\
& \sum_{j \in N} P_{0, j}^{r}=1 \quad \forall r \in R \\
& \sum_{j \in N} P_{j,(n+1)}^{r}=1 \quad \forall r \in R \\
& \sum_{i \in N} P_{i, h}^{r}-\sum_{j \in N} P_{h, j}^{r}=0 \quad \forall r \in R \\
& s_{i}^{r}+w_{i}^{r}+t_{i, j} \leq T W-30 \quad \forall r \in R \\
& w_{j}^{r}=\left(e_{j}^{r}-s_{j}^{r}\right)+T P K_{j}^{r} \quad \forall r \in R
\end{aligned}
$$


Regading the details of problem, each engineer is assigned a daily service at 5 jobs and earn 30 pounds per job. The wage for each engineer depedns on the number of thier daily jobs completed, but the total wage cost paid for the fixed number of job is the same for the company. Thus, the objective function can be formed as (1) to minimises the total of the travel cost, which includes the total of distances travelled by workers and the total travel cost varies in different modes of transportation. Constraints (2) are the binary constraints for variable $P_{i, j}^{r}$. Constraints (3) ensures that each job is visited only once. Constraints (4) - (5) show that each route begins and ends at the office. Constraint (6) confirms that each task location is visited and left while (7) guarantees that each job starts and terminates within a time window. Parking time is set as a constant value of 15 minutes while lunchtime is defined at 30 minutes. Constraints (8) estimates the working time of a task $w_{i}^{r}$ calculated by a finishing time $e_{i}^{r}$, starting time $s_{i}^{r}$, and parking time $T P K_{i}^{r}$ of task $j$. Note that in this study worker are homogenous. All engineers earn daily pay and $w_{j}^{r}$ is set as two constant values at 90 and 180 minutes depending on types of meter installation.

\subsection{A data set}

The scheduling environment dataset considered in this computational study is extracted from an interview with an engineer.

\begin{tabular}{|c|c|c|c|c|c|}
\hline Location & Postcode & Type & Location & Postcode & Type \\
\hline 1 & PA1 XXX & A & 14 & G52 XXX & A \\
\hline 2 & PA1 XXX & A & 15 & G52 XXX & B \\
\hline 3 & PA1 XXX & A & 16 & G52 XXX & A \\
\hline 4 & PA2 XXX & B & 17 & G52 XXX & B \\
\hline 5 & PA2 XXX & A & 18 & G81 XXX & A \\
\hline 6 & PA1 XXX & B & 19 & G81 XXX & A \\
\hline 7 & PA3 XXX & A & 20 & G14 XXX & A \\
\hline 8 & PA3 XXX & A & 21 & G11 XXX & A \\
\hline 9 & PA3 XXX & B & 22 & G11 XXX & B \\
\hline 10 & PA1 XXX & A & 23 & G11 XXX & A \\
\hline 11 & PA1 XXX & A & 24 & G3 XXX & A \\
\hline 12 & PA2 XXX & A & 25 & G3 XXX & A \\
\hline 13 & G52 XXX & A & 26 & G3 XXX & Office \\
\hline
\end{tabular}

Table I provides twenty-five locations of customers, who require meter installation, are assumed from real postcodes in council areas of Glasgow City and Renfrewshire, UK. The ratio of Type-A: Type-B is set as 3:1.

\subsection{Experimental design and development}

The proposed algorithms have been developed with $\mathrm{R}$ language on laptop Intel i7 systems with 16 GB of RAM, running at 2.8-3.8 GHz on Windows 10 64-bits OS. A list of abbreviations is explained as follows:

\section{HRT : Heuristic method \\ GDS : Greedy search \\ TGA : Three-step scheduling with GA \\ $H G A$ : Three-step scheduling with $G A$ with local search}

For the heurisric method (HRT), the structure of postal codes or postcodes in the UK, each postcode consists of two alphanumeric codes. The first made up of three characters to indicate the city or region, and the second made up of three characters to allocate streets, and sides of the road. For example, "PA1 2AP", the first code is "PA1" and the second is "2AP". The scheduler/planner enables to create a service schedule by filtering, and grouping of the first code in a job list, i.e., Route-1 contains first codes of "PA1" and "PA2", and Route-2 contains "PA3" and "PA4" and so on. This heuristic scheduling procedure is acceptable and effective for planning, but it uses the experience of the human scheduler for arranging jobs within same first postcodes.

For a greedy search $(G D S)$, this method makes the optimal choice at each step to explore the overall of the entire problem. This method starts by creating a list of daily jobs for all of the engineers. Then, applying the greedy search to find the nearest job location of each engineer at the office locations based on the OD-matrix. In each step, a selected job location is moved from the job list to a personal schedule of each engineer. The procedure repeats until all jobs are entirely assigned.

Regarding the method based-on the three-step scheduling approach, the procedure of these methods are provided in Algorithms 1. The difference between the TGA and the $H G A$ is the $T G A$ employs a GA only, but the $H G A$ applies both GA and a local search for arranging the service schedule. The CPU time of TGA should less than the $H G A$ as the structure of its algorithm is smaller. Table I shows the parameter values of the three-step scheduling approach for the experiment.

TABLE II. PARAMETER SETTING FOR TGA AND HGA

\begin{tabular}{l|c}
\multicolumn{1}{c}{ Parameters } & Level of parameters \\
\hline Crossover rate $\left(P_{c}\right)$ & 0.5 and 0.7 \\
Mutation rate $\left(P_{m}\right)$ & 0.001 and 0.1 \\
Size of population & 20 and 40 \\
Maximum iteration & 300 \\
Replication & 3
\end{tabular}




\subsection{Experimental results}

TABLE III. AVERAGE RESULTS OF EACH PROCEDURE

\begin{tabular}{|c|c|c|c|c|c|c|c|c|c|}
\hline \multirow{3}{*}{$\begin{array}{l}\text { Type } \\
\text { HRT }\end{array}$} & \multirow{3}{*}{$\begin{array}{c}\text { Pc } \\
\text { N/A }\end{array}$} & \multirow{3}{*}{$\begin{array}{l}\text { Pm } \\
\text { N/A }\end{array}$} & \multirow{3}{*}{$\begin{array}{c}\text { Size of } \\
\text { population } \\
\text { N/A }\end{array}$} & \multirow{3}{*}{$\begin{array}{c}\text { Average } \\
\text { Distance(km) } \\
185.1\end{array}$} & \multirow{3}{*}{$\begin{array}{l}\text { SD } \\
\text { N/A }\end{array}$} & \multirow{3}{*}{$\begin{array}{c}\text { Average } \\
\text { CPU time (s) } \\
\approx 600\end{array}$} & \multirow[t]{2}{*}{ SD } & \multicolumn{2}{|c|}{ Average } \\
\hline & & & & & & & & \multicolumn{2}{|c|}{ \%-Improvement } \\
\hline & & & & & & & N/A & N/A & $\mathrm{N} / \mathrm{A}$ \\
\hline GDS & N/A & N/A & N/A & 247.59 & N/A & 1 & N/A & -33.76 & N/A \\
\hline \multirow{6}{*}{ TGA } & 0.5 & 0.001 & 20 & 179.62 & \multirow{6}{*}{$\begin{array}{c}177.18 \\
\mathrm{SD}=6.55\end{array}$} & 1.53 & \multirow{6}{*}{$\begin{array}{c}1.9 \\
\mathrm{SD}=0.37\end{array}$} & 2.96 & \multirow{6}{*}{4.28} \\
\hline & 0.5 & 0.001 & 40 & 171.64 & & 2.32 & & 7.27 & \\
\hline & 0.7 & 0.001 & 20 & 186.29 & & 1.53 & & -0.64 & \\
\hline & 0.7 & 0.001 & 40 & 173.68 & & 2.32 & & 6.17 & \\
\hline & 0.7 & 0.1 & 20 & 176.01 & & 1.54 & & 4.91 & \\
\hline & 0.7 & 0.1 & 40 & 169.10 & & 2.24 & & 8.64 & \\
\hline \multirow{4}{*}{ HGA } & 0.7 & 0.001 & 20 & 166.58 & \multirow{4}{*}{$\begin{array}{c}174.23 \\
\mathrm{SD}=9.05\end{array}$} & 1.66 & \multirow{4}{*}{$\begin{array}{c}1.98 \\
\mathrm{SD}=0.37\end{array}$} & 10.01 & \multirow{4}{*}{5.87} \\
\hline & 0.7 & 0.001 & 40 & 179.79 & & 1.95 & & 2.87 & \\
\hline & 0.7 & 0.1 & 20 & 174.59 & & 1.72 & & 5.68 & \\
\hline & 0.7 & 0.1 & 40 & 165.74 & & 2.43 & & 10.46 & \\
\hline
\end{tabular}

\section{Dashboard \\ Traffic \\ Requests/sec}

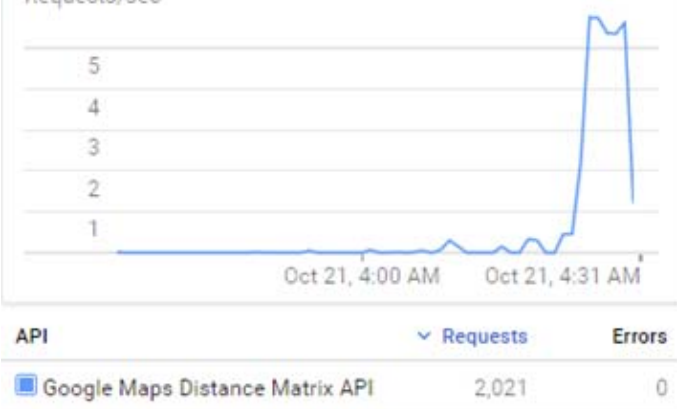

Dashboard

enable aPI

Traffic

Requests/sec

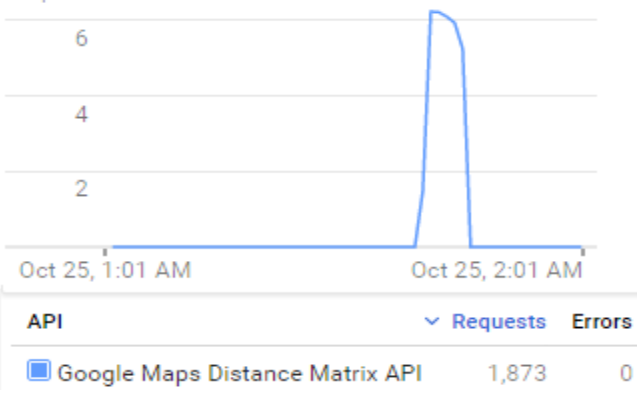

Fig. 5. Google Map API Dashboard
TABLE IV. AVERAGE TIME TO REQUEST DATA FROM GOOGLE

\begin{tabular}{ccc}
\hline \multicolumn{3}{c}{ Response time in second } \\
\hline Replication 1 & Replication 2 & Average \\
334.72 & 305.83 & 320.28 \\
\hline
\end{tabular}

$\begin{array}{rrr}\text { user } & \text { system elapsed } \\ 142.65 & 172.25 & 334.71\end{array}$

$\begin{array}{rrr}\text { user } & \text { system elapsed } \\ 137.39 & 157.09 & 305.83\end{array}$

Fig. 6. Computational times for obtaining data from Google Map (334.72 and $305.83 \mathrm{~s}$.)

As shown in table III, although the $H R T$ is a simple method, not needing to use any computers or even calculators for calculation, this original method shows an impressive total of service distance at $185.1 \mathrm{~km}$ for five engineers to offer the daily service. This method is used as a reference for other methods that we are discussed later on.

In contrast, using a simple heuristic algorithm, the GDS shows results at $247.59 \mathrm{~km}$., which is worse than the original method by 33.76 per cent; even it takes a minimum execution time just 1 second.

Regarding the GA-based approach, in overall, the $H G A$ method has slightly better results than the $T G A$ at the average $174.23 \mathrm{~km}$ and $177.18 \mathrm{~km}$ respectively. The CPU times of both methods are also barely a difference. Compared to the original, the average \%-improvement of the $H G A$ and the $T G A$ are 4.28 and 5.87 . 
In respect of time for obtaining data from Google for the GDS, TGA and HGA for practical use, fig. $\mathbf{5}$ shows the Google Map API dashboard while a connection between our scheduling programming and Google Map is connected. Fig. 6 provides computational times for obtaining data from Google Map for two instances at $334.72 * *$ and 305.83 seconds respectively. The average time is 320.28 seconds for 26 actual locations as shown in table IV. It can be seen that the time for obtaining Google's data is significantly higher than the CPU time spend for our proposed methods. Thus, obtaining time from Google is also taken into account in the model. Concerning to the worst case of our proposed methods, the actual computational time must include the worst CPU time of the proposed method and the worst for obtaining Google's data which is approximate at $2.43 *(\mathrm{HGA})+334.72 * *\left(1^{\text {st }}\right.$ for obtaining Google's data) $=337.15$ seconds or 5.62 minutes.

\section{CONCLUSION}

This paper introduces a three-step scheduling, a generic framework for solving the workforce planning problems of the EMI service. A simulated data set generated from the interview is used to measure the performance of the proposed framework.

Our empirical study reveals that the proposed technique is able to explore the shortest service path with the total distance travelled by engineers, compared to the original.

This paper provides a potential basis for future research. We will point to the stochastic model for the EMI service problem that the operational time can vary between 90 to 150 minutes. On the one hand, we can continue to apply the framework for a similar scheduling problem of mentioned in this paper.

\section{ACKNOWLEDGMENTS}

This publication was made possible by grants from the Innovate UK project - Personalised Service Assistant for SPAD Journey Planner to carry out this research.

\section{REFERENCES}

[1] G. D. Service. "Smart meters: a guide," 04 May, 2019; www.gov.uk/guidance/smart-meters-how-they-work.

[2] P. De Bruecker, J. Van den Bergh, J. Beliën, and E. Demeulemeester, "Workforce planning incorporating skills: State of the art," European Journal of Operational Research, vol. 243, no. 1, pp. 1-16, 5/16/, 2015.

[3] S. Carlos, A. Sánchez, and S. Martorell, "Model to study the effect of workforce on a safety equipment and its optimization," Mathematical and Computer Modelling, vol. 54, no. 7-8, pp. 1808-1812, 2011.

[4] A. Corominas, A. Lusa, and J. Olivella, "A detailed workforce planning model including non-linear dependence of capacity on the size of the staff and cash management," European Journal of Operational Research, vol. 216, no. 2, pp. 445-458, 2012.

[5] T. Majozi, and X. Zhu, "A combined fuzzy set theory and MILP approach in integration of planning and scheduling of batch plants-Personnel evaluation and allocation," Computers \& chemical engineering, vol. 29, no. 9, pp. 2029-2047, 2005.

[6] E. K. Burke, P. De Causmaecker, G. V. Berghe, and H. Van Landeghem, "The state of the art of nurse rostering," Journal of scheduling, vol. 7, no. 6, pp. 441-499, 2004.

[7] S. Irnich, P. Toth, and D. Vigo, "Chapter 1: The family of vehicle routing problems," Vehicle Routing: Problems, Methods, and Applications, Second Edition, pp. 1-33: SIAM, 2014.
[8] M. Desrochers, J. Desrosiers, and M. Solomon, "A new optimization algorithm for the vehicle routing problem with time windows," Operations research, vol. 40, no. 2, pp. 342-354, 1992.

[9] A. E. Carter, and C. T. Ragsdale, "A new approach to solving the multiple traveling salesperson problem using genetic algorithms," European Journal of Operational Research, vol. 175, no. 1, pp. 246-257, 2006.

[10] M. Gendreau, G. Ghiani, and E. Guerriero, "Time-dependent routing problems: A review," Computers \& Operations Research, vol. 64, pp. 189-197, 2015/12/01/, 2015. 